\title{
Blindleistungsbereitstellung aus Flächenverteilnetzen - praktische Umsetzung in einem Feldtest
}

\author{
Marcus Kreutziger (D) - Sebastian Wende-von-Berg · Sebastian Krahmer · Peter Schegner
}

Eingegangen: 17. September 2021 / Angenommen: 30. November 2021 / Online publiziert: 18. Februar 2022

(c) Der/die Autor(en) 2022

\begin{abstract}
Zusammenfassung Im Rahmen des Beitrags sollen das Potenzial der Blindleistungsbereitstellung und mögliche Regelungskonzepte im Kontext von Redispatch 2.0 dargestellt werden. Ein umfangreicher Feldtest zeigt das Zusammenspiel von Übertragungsund Verteilnetzbetreibern bezüglich einer spannungsebenenübergreifenden Blindleistungsregelung auf. Neben der Konzeption und Entwicklung aller Systemkomponenten wurden die Funktionalität einer aktiven Blindleistungsregelung und deren Wirkung auf den realen Netzbetrieb evaluiert.
\end{abstract}

Schlüsselwörter Blindleistungsbereitstellung • Spannungshaltung · Verteilnetz · Übertragungsnetz • Dezentrale Erzeugungsanlage $\cdot$ Redispatch 2.0

\section{Provision of reactive power from wide area distribution grids - practical implementation in a field test}

Abstract This article presents the potential of reactive power provision and control in the context of Redispatch 2.0. An extensive field test shows the interaction of transmission and distribution system operators with regard to a reactive power control across several voltage levels. In addition to the design and development of all system components, the functionality of an active control of reactive power and its effect on the real grid area were evaluated.

\footnotetext{
M. Kreutziger $(\bowtie) \cdot$ S. Krahmer $\cdot$ P. Schegner

Technische Universität Dresden,

Mommsenstraße 10, 01069 Dresden, Deutschland

marcus.kreutziger@tu-dresden.de

S. Wende-von-Berg

Fraunhofer IEE, Kassel, Deutschland
}

Keywords Reactive power supply · Voltage maintenance - Distribution grid · Transmission grid . Distributed generator · Redispatch 2.0

\section{Einleitung}

Durch die kontinuierliche Reduzierung konventioneller Kraftwerksleistung und der Zunahme regenerativer Energieerzeugung mit der einhergehenden Dezentralisierung, kommt vor allem regionalen Verteilnetzen eine steigende Bedeutung bei der Bereitstellung von Systemdienstleistungen $\mathrm{zu}$.

Das seit 19. Mai 2019 geltende Netzausbaubeschleunigungsgesetz (NABEG) beschreibt dabei den Umgang mit Netzengpässen. Darin enthaltene Vorgaben sind bis 1. Oktober 2021 durch die Netzbetreiber umzusetzen. Es soll ein einheitliches RedispatchVorgehen nach $\$ \S 13$, 13a und 14 Energiewirtschaftsgesetz (EnWG) etabliert werden. Kernbestand ist die Fernsteuerbarkeit von dezentralen Energieerzeugern ab $100 \mathrm{~kW}$ durch den Netzbetreiber und somit deren Beteiligung am s.g. Redispatch 2.0.

Grundvoraussetzung ist dabei eine intensive $\mathrm{Zu}$ sammenarbeit der Netzbetreiber bei der Behebung von Netzengpässen mit der Prämisse möglichst geringer Gesamtkosten über alle Netzebenen und unter Einhaltung der Netz- und Versorgungssicherheit.

Diese Vorgaben fokussieren die Regelung strombedingter Engpässe, welche durch regionale Disbalancen von Erzeugung und Verbrauch auftreten. Projekte wie IMOWEN [1] und EU-SysFlex [2] liefern hierfür die ersten Lösungen.

In einem nächsten Schritt gilt es das Konzept auf die Bewirtschaftung spannungsbedingter Engpässen zu erweitern. Das Projekt SysDL 2.0 lieferte hierfür bereits grundlegende Lösungsansätze um ein spannungsebenenübergreifendes Blindleistungsmanagement durchzuführen. Von der Konzipierung der erfor- 
derlichen Anwendungsfälle über die Entwicklung der Algorithmen und Diensteplattform bis hin zur praktischen Erprobung in realen Netzgebieten zeigte ein vielversprechendes Pilotsystem mögliche Lösungen [3].

Dieser Beitrag zeigt die Umsetzung und Auswertung des im Projekt entwickelten Systems im Kontext der aktuellen Entwicklungen. Die Umsetzung erfolgte dabei in Zusammenarbeit der TU Dresden, des Fraunhofer IEE, der Siemens AG, zweier Verteilnetzbetreiber (Mitnetz Strom mbH, SachsenNetze GmbH) und einem Übertragungsnetzbetreiber (50 Hertz Transmission $\mathrm{GmbH}$ ).

\section{Technisch-wissenschaftlicher Hintergrund}

\section{Anwendungsfälle und Optimierungsansatz}

Um allgemeine Anforderungen für ein spannungsebenenübergreifendes Blindleistungsmanagement ableiten zu können, wurden zunächst sechs Anwendungsfälle in Anlehnung an [4] durch das Projektkonsortium identifiziert [5]. Die folgenden Anwendungsfälle sind dabei auch Grundlage für die Umsetzung des Redispatch 2.0 Ansatzes:

- Potenziale der Spannungsbänder am Netzverknüpfungspunkt (NVP)

- Blindleistungspotenziale am NVP

- Einstellung von Blindleistungswerten am NVP

- Minimierung der Netzverluste im Verteilnetz

Die Umsetzung durchlief die komplette Kette von der Konzeption, über die wissenschaftliche Untersuchung bis hin zur praktischen Erprobung in einem Feldtest.

Die Anwendungsfälle der lokalen Spannungshaltung sowohl im Verteilnetz als auch des lokalen Engpassmanagements werden als Randbedingungen für den Optimierungsalgorithmus interpretiert. Voraussetzung für die Durchführung des Feldtests ist ein Netz mit ausreichend Reserven hinsichtlich der Leitungsbelastung sowie der Spannungsgrenzwerte.

Auf dieser Grundlage wurden Netzbetriebsführungsalgorithmen entwickelt, welche sowohl die Anforderungen einzelner aber auch eine komplexere Kombinationen verschiedener Anwendungsfälle erfüllen [6].

\section{Systemansatz zum Blindleistungsmanagement}

Bei der Umsetzung des Systemansatzes wurden sowohl Algorithmen zur Topologieerkennung, Netzzustandsschätzung, Prognose und Blindleistungsoptimierung entwickelt bzw. adaptiert [6-8]. Zur Darstellung aller notwendigen Informationen wurde eine grafische Benutzeroberfläche entwickelt und in $\mathrm{Zu}$ sammenarbeit mit den Netzbetreibern auf Praktikabilität getestet [3].

Das System durchläuft im Betrieb folgende Einzelprozesse:
1. Übertragung und Speicherung der Daten des Netzbetreibers im CIM-Format [9]

2. Erstellung des Netzmodells auf Basis der Messdaten

3. Ausführung der Zustandsschätzung

4. Abruf der Prognosedaten

5. Ausführung der Optimierung für Ist-Zustand und Prognosezeitraum

6. Eingabe der optimierten Blindleistungswerte

7. Monitoring

Sowohl die Optimierungsergebnisse als auch die Kontrolle des resultierenden Zustandes werden dem Systemoperator über die grafische Nutzeroberfläche zur Verfügung gestellt.

\section{Vorbereitung des Feldtests}

\section{Anforderungen und Konzeption}

Da der praktische Funktionstest aller Komponenten in der realen Netzumgebung unter regulären Betriebsbedingungen durchgeführt werden sollte, mussten spezielle Anforderungen aber auch Randbedingungen formuliert werden. Priorität war es, Einschränkungen im regulären Netzbetrieb zu vermeiden. Weiterhin sollte das SysDL-System Zugriff auf alle nötigen Daten des Leitsystems haben, allerdings nicht direkt darin integriert sein. Zudem sollte das System im Rahmen des Feldtests keine direkte Anlagensteuerung vornehmen können.

Dies hatte zur Folge, dass Schnittstellen zum automatischen Datenaustausch implementiert wurden, die Anlagensteuerung jedoch durch den Systemführer manuell eingegeben werden musste. Dies schränkt zum einen den zeitlichen Umfang eines aktiven Feldtests, als auch den Umfang der zu steuernden Anlagenanzahl ein. Der Feldtest wurde in zwei Abschnitte - passiv und aktiv - unterteilt. Während des passiven Feldtests, wurde keine Anlagensteuerung durchgeführt, jedoch alle Komponenten und Berechnungen des SysDL-Systems plausibilisiert und validiert. Anschließend wurden innerhalb des aktiven Feldtests, Anlagensteuerungen im laufenden Netzbetrieb vorgenommen.

\section{Auswahl der Netzgebiete und Anlagen}

Um den Feldtest durchführen zu können, waren Netzgebiete mit technisch geeigneten Erzeugungsanlagen und einer ausreichenden installierten Leistung erforderlich. Die Netzgebiete der beteiligten Netzbetreiber wurden entsprechend analysiert, anhand der geforderten Kriterien bewertet und jeweils eine Netzgruppe für den Feldtest ausgewählt. Abb. 1 zeigt eine Übersicht der Netzgebiete.

Im Fokus standen Anlagen, welche nach 2014 in Betrieb gegangen sind, da diese nach VDE-ARN4120 [10] einen erweiterten Blindleistungsstellbereich haben und durch den Netzbetreiber fernsteu- 


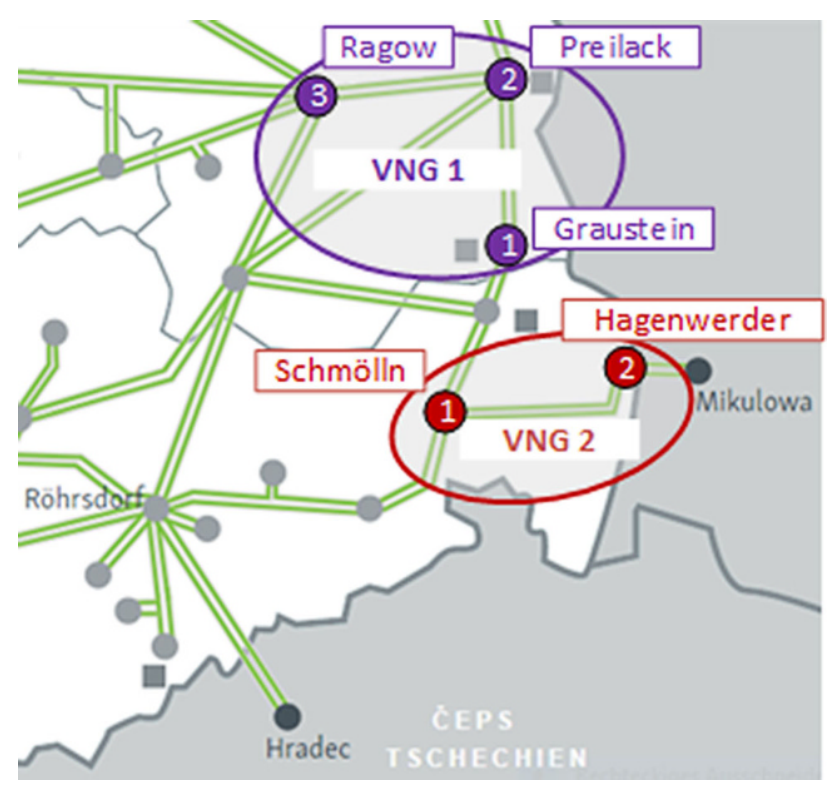

Abb. 1 Netzgebiete im Projekt. (Nach [3])

erbar sein müssen. Weitere Untersuchungen wurden hinsichtlich einer möglichst hohen Blindleistungsverfügbarkeit im Jahr sowie einer hohen Sensitivität bezüglich der beschriebenen Anwendungsfälle durchgeführt [11]. Der Feldtest wurde in jeweils einer Verteilnetzgruppe (VNG) der beiden Verteilnetzbetreiber durchgeführt.

VNG 1 ist über drei Netzverknüpfungspunkte mit dem Übertragungsnetz verbunden. Im verbundenen Übertragungsnetz sind dabei hohe Leistungen durch konventionelle Kraftwerke angeschlossen, was eine hohe Spannungsstabilität für den Feldtest zur Folge hat. In der VNG 1 sind insgesamt 40 Erzeugungsanlagen mit einer installierten Leistung von etwa 1700 MVA angeschlossen, wovon 13 Anlagen steuerbar waren.

VNG 2 ist über zwei Netzverknüpfungspunkte mit dem Übertragungsnetz verbunden. In diesem Netzgebiet wurden drei Windparks mit einer installierten Leistung von 32 MVA für eine flexible Steuerung nachgerüstet.

\section{Planung des Feldtests}

Um den regulären Netzbetrieb so wenig wie möglich zu beeinflussen und unvorhergesehene Auswirkungen durch die Steuerung zu minimieren, wurde der Feldtest in drei Phasen unterteilt.

Phase 1 Das im Projekt entwickelte SysDL-System wurde parallel zum Leitsystem aufgebaut. Anschließend wurden, ohne Steuerungen vorzunehmen, alle Funktionalitäten und Berechnungen getestet. Dies beinhaltete die Erstellung der Netzmodelle auf Basis der Live-Netzdaten des Leitsystems, die Netzzustandsschätzung, die Berechnung der Blindleistungsbzw. Spannungspotenziale sowie die Optimierung hinsichtlich der Verlustminimierung. Die Ergebnisse wurden anschließend umfassend geprüft und ausgewertet.

Phase 2 Es wurde eine Steuerung einer geringen Anzahl an Erzeugungsanlagen ohne Beteiligung des ÜNB durchgeführt.

Phase 3 In der letzten Phase des Feldtests wurde das Zusammenspiel aller Akteure und Komponenten geprüft. Durch den ÜNB wurde ein Blindleistungssollwert an einem NVP vorgegeben, die Optimierung durch das SysDL-System durchgeführt und die Anlagensollwerte durch den Systemführer freigegeben. Die Zahl der zu steuernden Anlagen wurde auf eine manuell beherrschbare Anzahl begrenzt.

\section{Durchführung des Feldtests}

Die Durchführung der einzelnen Phasen des Feldtests wird anhand von VNG 1 beschrieben. Hier können alle wesentlichen Funktionalitäten und Erkenntnisse abgeleitet werden. Ergänzend werden kurz die Erfahrungen aus VNG 2 geschildert.

\section{Phase 1 - Parallelbetrieb}

Die erste Stufe des Feldtests erfolgte über einen Zeitraum von neun Monaten, in denen zunächst der Datenaustausch zwischen dem SysDL-System und allen Datenquellen getestet und Schnittstellenfehler behoben wurden. Die Funktion der einzelnen Algorithmen wurden evaluiert und entsprechende Weiterentwicklungen durchgeführt. Zudem wurde die Bedienoberfläche und Darstellung der Ergebnisse im praktischen Einsatz der Netzbetreiber geprüft und angepasst. Nachdem das Gesamtsystem eine ausreichende funktionale Stabilität erreicht hatte, wurde mit Phase 2 des Feldtests begonnen.

Folgend sollen zunächst die Ergebnisse der einzelnen Funktionalitäten aus Phase 1 dargestellt werden.

\section{Datenauskopplung/Erstellung Netzmodell}

Die notwendigen Daten für den Echtzeitbetrieb wurden zyklisch aus den Netzleitwarten mithilfe von CIM-Schnittstellen und CSV-Exporten bereitgestellt und über eine Import-Schnittstellen des SysDL-Systems verarbeitet.

Auf Basis der Topologie- und Betriebsmitteldaten wurde ein Netzmodell erstellt. Hierfür wurde das Open Source Netzberechnungs- und Analyseframework pandapower [12] verwendet. Messwerte der Verbrauchs- und Erzeugungsanlagen wurden je nach Netzbetreiber entweder direkt in dem CIM-Model bereitgestellt oder über eine separate CSV-Tabelle nachgeliefert. Auf dieser Basis konnte ein initialer Lastfluss berechnet werden, um den Netzzustand auf Rechenfähigkeit hin zu prüfen. Zusätzliche Span- 


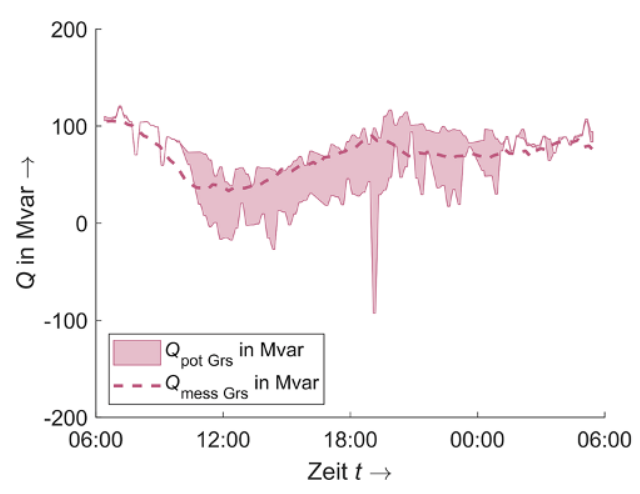

Abb. 2 Verlauf des Blindleistungspotenzials am NVP Graustein der VNG 1 über einen Tag

nungs- und Leistungsflussmessungen wurden für die Zustandsschätzung verwendet.

Neben den aktuellen Messungen wurden Prognosen aller relevanten Erzeugungs- und Verbrauchsanlagen durch den VNB bereitgestellt. Mithilfe dieser Daten wurden dann für jeden Zeitpunkt im Prognosezeitraum (vier Stunden mit einer 15-min-Auflösung) jeweils ein „Prognosenetz“ erstellt und ein Lastfluss durchgeführt.

\section{Netzzustandsschätzung}

Im nächsten Schritt wurde die Zustandsschätzung im realen Netzbetrieb durchgeführt. Ziel war zunächst ein Abgleich der Zustandsschätzung mit dem Netzabbild im Leitsystem der Netzbetreiber.

Optimierungsziel der Zustandsschätzung war die Minimierung der Abweichungen bezüglich der Leistungsflüsse im Verteilnetz. Dabei war festzustellen, dass bei der Nachbildung der 380-kV-Leitungen die größten Abweichungen auftraten, was auf die Randnetzreduzierung zurückzuführen ist. Die größten Abweichungen innerhalb von VNG 1 traten folglich auf den Verbindungsleitungen zum Übertragungsnetz auf. Die Abweichungen der 75-\%-Perzentile über allen Leitungen lag dabei bei $\Delta P_{75} \mathrm{Ltg}=+15 /-17 \mathrm{MW}$ und $\Delta Q_{75 \mathrm{Ltg}}=+12 /-21 \mathrm{Mvar}$.

Für die nachfolgende Optimierung der Blindleistungsbereitstellung an den NVP, war deren genaue Abbildung notwendig. Die Auswertung der 75-\%Perzentile aller Messwerte der NVP-Summenleistung zeigte dabei Abweichungen von etwa $\Delta P_{75 \mathrm{NVP}}=$ $+18 /-25 \mathrm{MW}$ und $\Delta Q_{75 \mathrm{NVP}}=+6 /-22 \mathrm{Mvar}$. Die Mediane lagen bei $\Delta P_{\text {Med NVP }}=+5 /-5 \mathrm{MW}$ und $\Delta Q_{\text {Med NVP }}=$ $-5 /-14$ Mvar. Setzt man diese Werte in Relation zur übertragbaren Leistung mit jeweils zwei/drei Transformatoren und somit 600-900 MVA Bemessungsleistung je NVP, lagen die beschriebenen maximalen Abweichungen unterhalb von 5\%. Dies bedeutet, dass die größte Anzahl der Abweichungen im Bereich der Messungenauigkeit der Leistungsmessungen von $3 \%$ lag.

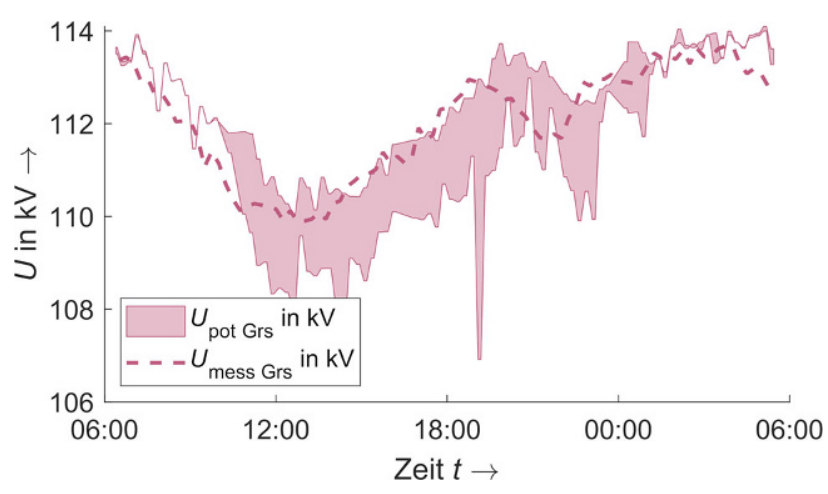

Abb. 3 Verlauf des Spannungsstellpotenzials am NVP Graustein der VNG 1 über einen Tag

\section{Blindleistungspotenziale}

Ein weiteres Ziel war die Ermittlung und Bewertung der Potenziale für die beschriebenen Anwendungsfälle. Es wurden Berechnungen für den gesamten Zeitraum durchgeführt, ausgewertet und notwendige Anpassungen der Algorithmen vorgenommen. Folgend soll die Funktionalität anhand eines Beispieltages in VNG 1 durchgeführt werden.

In den Darstellungen wird eine Blindleistungsbereitstellung zum Übertragungsnetz mit positivem Vorzeichen dargestellt, es erfolgt eine Spannungsanhebung. Ein Blindleistungsbezug führt zur Spannungsabsenkung und wird mit negativen Vorzeichen angegeben.

Abb. 2 zeigt den Bereich, in dem es möglich ist Blindleistung an einem NVP in VNG 1 bereitzustellen. Der maximale Blindleistungsstellbreich liegt bei-95 bis 110 Mvar.

\section{Spannungspotenziale}

Bei der Auswertung der Spannungspotenziale wurde eine Unterscheidung in zwei unterschiedliche Anwendungen vorgenommen. Im ersten Fall wurde das Spannungsstellpotenzial, resultierend aus der Blindleistungsregelung der Anlagen im Verteilnetz bestimmt. (vgl. Abb. 3.). Die maximale Beeinflussbarkeit der Spannung an diesem NVP liegt bei 2 bis 2,5 kV.

Demgegenüber ist es für den ÜNB wichtig, welche maximale Spannung er dem VNB am NVP zumu-

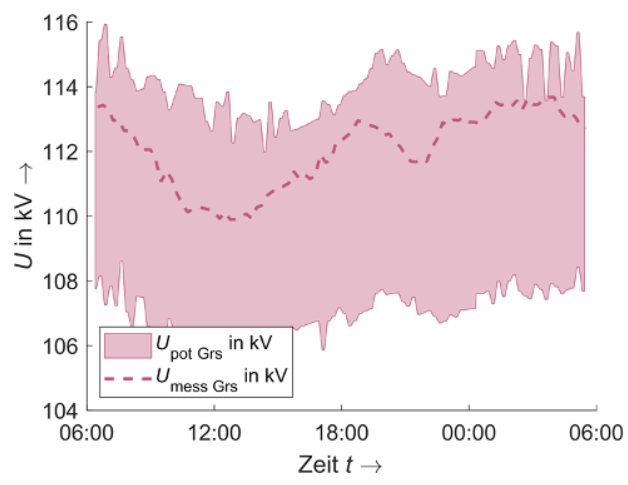

Abb. 4 Verlauf der zulässigen Spannungsgrenzen am NVP Graustein über einen Tag 

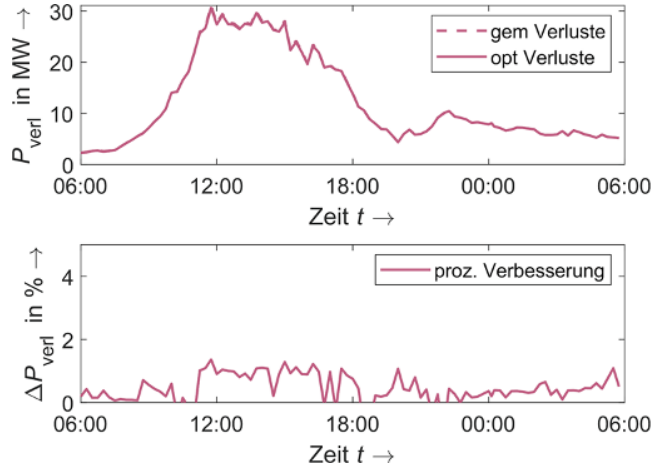

Abb. 5 Verlauf der Verlustoptimierung

ten kann, ohne Grenzwertverletzungen im Verteilnetz hervorzurufen. Das Potenzial in Abb. 4. ist dabei auf der 110-kV-Seite des NVP angegeben. Der ÜNB hat dabei ein Potenzial von 4 bis $5 \mathrm{kV}$ zur Verfügung. Der aktuelle Spannungsmesswert ist auch hier im oberen Bereich des Potenzials zu finden.

\section{Optimierung der Leitungsverluste}

Im Gegensatz zu den signifikanten Potenzialen der Blindleistungs- und Spannungsbeeinflussung, ist die Verlustoptimierung begrenzt. Bereits in Abb. 3. ist zu erkennen, dass die Spannung im oberen Potenzialbereich liegt. Da eine höhere Spannung im Allgemeinen zu einer Verlustreduzierung führt, ist das Potenzial für eine weitere Reduzierung gering. Abb. 5. zeigt den Verlauf der Verluste und das Optimierungspotenzial eines Tages. Die Verluste steigen dabei über den Tagesverlauf an und fallen nachts wieder ab. Das Potenzial einer Verlustoptimierung liegt bei maximal 1,5\%. Dieses Potenzial lässt sich mit einer höheren Anzahl regelbarer Anlagen steigern [6].

\section{Phase 2 - Steuerung von Einzelanlagen}

Um das Verhalten von Anlagen und Netz auf die Blindleistungsregelung zu beobachten, wurden in Phase 2 des Feldtests jeweils nur wenige Anlagen gesteuert. In VNG 1 wurde der Test aufgrund der Wetterlagen mit wenig Wind mit zwei aktiv steuerbaren PV-Anlagen und hoher installierter Leistung durchgeführt. Beide Anlagen sind in unmittelbarer Nähe des NVP Graustein angeschlossen.

Aus dem Blindleistungspotenzial (analog zu Abb. 2.) wurde zunächst ein möglicher Sollwert ermittelt. Dieser Sollwerte entsprach mit -46 Mvar dem zu diesem Zeitpunkt maximal ermittelten Potenzial. Der Blindleistungswert lag zu Beginn bei -58 Mvar.

Um eine zu starke Spannungsänderung am Anschlusstransformator der Anlagen und somit eine Schutzauslösung zu verhindern, war die Änderung der Blindleistung auf 3 Mvar je $2 \mathrm{~min}$ pro Anlage begrenzt. Dies hatte zur Folge, dass 4 Steuerungsschritte je Optimierungszyklus notwendig waren [10]. Die Eingabe der Sollwerte erfolgte manuell durch den

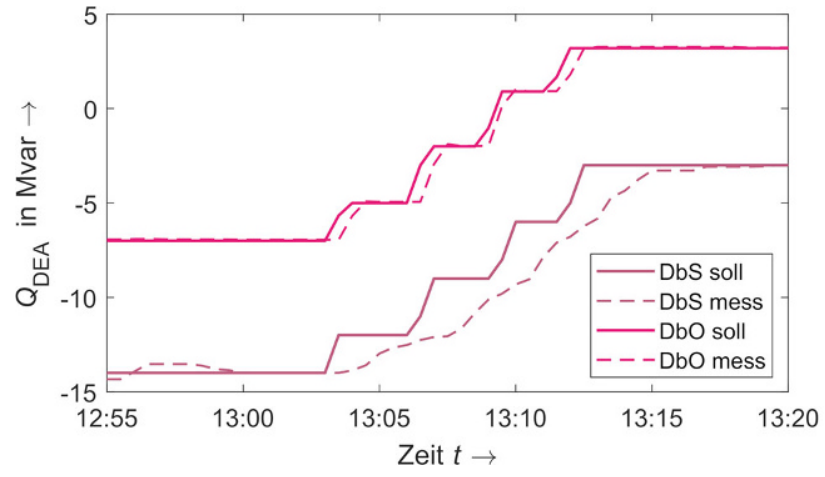

Abb. 6 Verlauf der Sollwertvorgabe und Anlagenregelung

Operator direkt über das Leitsystem. Während der Optimierung wurden alle notwendigen Netzrestriktionen (Spannungsbänder, maximale Leitungsbelastung, ( $n$ 1)-Kriterium) eingehalten.

Abb. 6. zeigt den Verlauf der Sollwerteingabe und die jeweils gemessenen Blindleistungswerte der Anlagen. Zu erkennen ist, dass Anlage DbS deutlich schneller dem Sollwert folgt als Anlage DbO. Beide Anlagen erreichen aber exakt den vorgegebenen Blindleistungssollwert. Auch am NVP Graustein wurde der Sollwert mit einer geringen Abweichung erreicht. In Abb. 7 ist auch zu erkennen, dass die beiden Anlagen in Summe (DEA Db) deutlich mehr Blindleistung bereitstellen, als deren Wirkung am NVP zu sehen ist.

Phase 2 des Feldtests in VNG 2 wurde mit drei Windparks und einer installierten Leistung von $32 \mathrm{MW}$ durchgeführt. Während des Testzeitraums, wurden die Anlagen hier von einem maximalen Blindleistungsbezug auf maximale Blindleistungsbereitstellung geregelt. Dies führte nach kurzer Zeit zu einer Schutzauslösung am Abgang eines Windparks. Der Feldtest musste daraufhin beendet werden.

Die anschließende Untersuchung ergab, dass die Schwelle zur Schutzauslösung, statt üblicherweise 1,1 p.u. auf 1,07 p.u. parametriert wurde. In Folge der übererregten Blindleistungsregelung während des Feldtests wurde die Grenze der Schutzauslösung kurzzeitig überschritten. Da sich die Schutzeinrichtung des Pilot-Umspannwerks im Eigentum eines Kunden

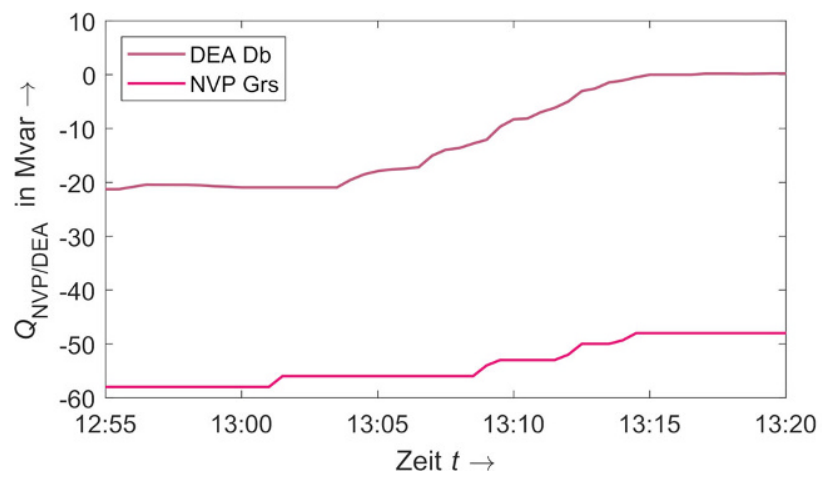

Abb. 7 Vergleich der geregelten Blindleistung beider Anlagen und Wirkung am NVP Graustein 
befindet, ist dieser für dessen Parametrierung verantwortlich. Auf Phase 3 des Feldtests wurde in diesem Netzgebiet verzichtet.

\section{Weitere Ergebnisse}

Vor allem durch Bedienung zweier parallellaufender Systeme (Leit- und SysDl-System) wurden Grenzen einer umfangreichen Phase 3 des Feldtests festgestellt. Durch die manuelle Eingabe der Sollwerte ist eine gleichzeitige Ansteuerung von mehr als 5-8 Anlagen nicht sinnvoll umsetzbar.

\section{Phase 3 - Komplextest}

Phase 3 des Feldtests in VNG 1 sollte mit einer ausreichenden Anzahl von Anlagen und in Zusammenarbeit mit dem ÜNB durchgeführt werden. Weiterhin wurde darauf geachtet, dass am Tag des Feldtests ausreichend Wind sowie Sonneneinstrahlung prognostiziert wurde.

Zunächst wurden 13 Anlagen, über das gesamte Netzgebiet verteilt, in die Potenzialermittlung sowie die aktive Blindleistungssteuerung aufgenommen. $\mathrm{Zu}-$ sätzlich zu den zwei PV-Anlagen aus Phase 1 wurden 11 Windenergieanlagen einbezogen. Die Blindleistungsbereitstellung sollte wieder am NVP Graustein erfolgen.

In Abstimmung mit dem ÜNB wurden anschließend 0Mvar und somit ein blindleistungsneutraler Betrieb an diesem NVP vereinbart. Entsprechend der Zielgröße wurden die Sollwerte für die regelbaren Anlagen berechnet und durch den Systemoperator manuell eingegeben (analog zu Phase 2).

Im Laufe des Feldtests zeigte sich, dass eine manuelle Eingabe der Sollwerte für 13 Anlagen nicht möglich war. Aus diesem Grund wurden lediglich die fünf Anlagen mit dem höchsten Einfluss gesteuert. Dies hatte zur Folge, dass ein geringerer Einfluss auf die Blindleistung am NVP Graustein je Optimierungsschritt erfolgen konnte und Abweichungen zum errechneten Verlauf nicht vermeidbar waren.

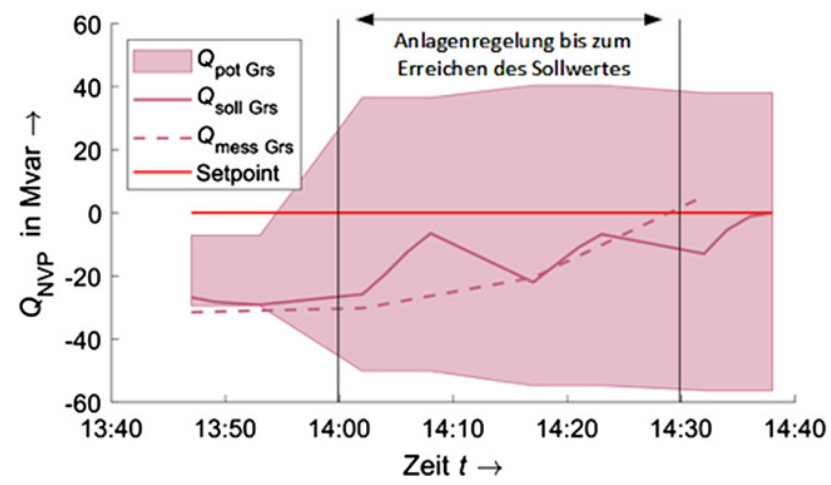

Abb. 8 Verlauf der Optimierung im aktiven Feldtest an NVP Graustein
Der detaillierte Ablauf ist folgend beschrieben und in Abb. 8. dargestellt. Abb. 9 zeigt zusätzlich den Verlauf der Blindleistungsregelung der fünf Anlagen.

\section{Detaillierter Ablauf}

13:45 Uhr Zielwert der Blindleistung am NVP wurde auf 0 Mvar gesetzt.

13:55 Uhr Die ausgewählten 5 Anlagen wurden zur Steuerung freigeschaltet, wodurch sich das Blindleistungspotenzial ergab. Die ersten Sollwerte werden durch die Optimierung berechnet.

14:00 Uhr Die ersten Blindleistungssollwerte wurden alle 2 min an die Anlagen übergeben. Zu erkennen ist, dass die Blindleistung am NVP dem Sollwert verzögert folgt.

14:10 Uhr Die Abweichung zum vorhergehenden Sollwert wird durch den Algorithmus erkannt, wodurch der erste Einstellschritt des nächsten Zyklus nahe dem aktuellen Messwert startet. Die Reaktion am NVP erfolgt erneut mit Verzögerung und geringerer Wirkung.

14:20 Uhr Erneut führt der Algorithmus eine Korrektur infolge der Abweichungen zum Vorgabewert durch. Der Sollwert am NVP wird erreicht.

\section{Weitere technische Ergebnisse und „Lessons Learned"}

Während des Feldtests wurden immer wieder zeitliche, aber auch numerische Abweichungen von Sollund Messwert festgestellt. Die Abweichungen sind dabei auf eine Vielzahl von Gründen zurückzuführen. Die beschriebene reduzierte Wirkung je Optimierungszyklus am NVP ist auf die Steuerung der 5 wirkungsvollsten Anlagen anstatt 13 Anlagen gesamt zurückzuführen. Die Eingabe einer höheren Anzahl von Sollwerten war mittels manueller Eingabe nicht durchführbar.

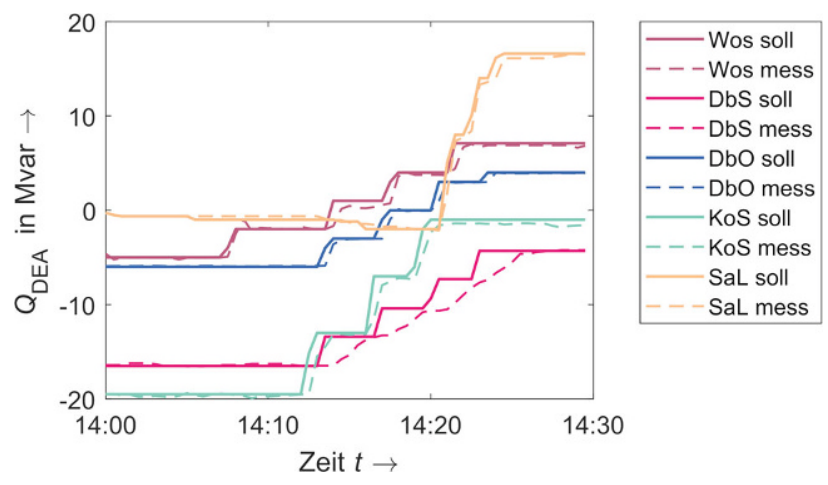

Abb. 9 Verlauf der Blindleistung ausgewählter Anlagen im aktiven Feldtest 
Der Zeitverzug in der Prozesskette trat in erster Linie durch die Messwert- und Netzdatenaufnahme, Berechnungszeit der Optimierungsergebnisse sowie der manuellen Sollwerteingabe auf. Ein weiterer Einfluss ist durch die benötigte Zeit der Anlagenregelung begründet. In Abb. 9 ist dieses Verhalten bei nahezu allen Anlagen zu beobachten, wobei die Reaktions- und Regelzeit deutliche Unterschiede aufweisen. So folgen einige Anlagen sehr schnell der Sollwertvorgabe, während andere Anlagen bis zu zwei Minuten benötigen.

Weiterhin konnte beobachtet werden, dass eine Anlage ein falsches Regelverhalten zeigte. Diese Anlage stellte nahezu den negativen Wert des Sollwertes ein und behält diesen trotz wiederholender Sollwertvorgabe bei. Eine nachträgliche Prüfung stellte fest, dass die Blindleistungsmessung an der Anlage ein falsches Vorzeichen ausgegeben hat.

\section{Zusammenfassung}

Im Rahmen des Projektes SysDL 2.0 wurde das Zusammenspiel von ÜNB und VNB im Rahmen der Blindleistungs- und Spannungsregelung durch VNGs in einem Feldtest evaluiert. Der Feldtest konnte die Funktionalität und das Zusammenspiel aller Akteure und Systemkomponenten nachweisen. Es konnten die Potenziale der Blindleistungsregelung auf die NVPs über einen längeren Zeitraum aufgezeigt werden. Dabei sind diese Potenziale in erster Linie von der Erzeugerstruktur (Anschlussjahr und -ort) und deren installierter Leistung abhängig.

Weiterhin konnte die vollständige Funktionalität einer aktiven Blindleistungsregelung und deren entsprechende Wirkung auf zwei reale Netzgebiete bestätigt werden. Mit Hilfe von aktiver Blindleistungsregelung können sowohl die Blindleistung am NVP zum Übertragungsnetz als auch der Blindleistungshaushalt bzw. die Spannungsbänder im Verteilnetz signifikant beeinflusst werden. Das Konzept zeigt somit das Potenzial der Blindleistungsregelung im Kontext von Redispatch 2.0 auf.

Um ein solches System automatisiert in den Netzbetrieb zu integrieren, gibt es aktuell jedoch noch einige Herausforderungen zu lösen. Vor allem im Bereich der Datenaufbereitung, Zustandsschätzung und Optimierung sind Potenziale hinsichtlich schnellerer Prozesszeiten, sowie der Erkennung von unplausiblen Messwerten und Netzzuständen vorhanden. Weiterhin ist es unabdingbar die genaue Konfiguration jeder Anlage zu kennen. Dies beginnt bei der installierten Leistung und dem Blindleistungsstellbereich, erstreckt sich über eine fehlerfreie Messung der aktuellen Größen bis hin zur Parametrierung der Schutzgeräte. Für alle Bereiche gibt es Normen bzw. Anwenderregeln. Die Anwendung dieser muss allerdings sichergestellt werden.

Funding Open Access funding enabled and organized by Projekt DEAL.
Open Access Dieser Artikel wird unter der Creative Commons Namensnennung 4.0 International Lizenz veröffentlicht, welche die Nutzung, Vervielfältigung, Bearbeitung, Verbreitung und Wiedergabe in jeglichem Medium und Format erlaubt, sofern Sie den/die ursprünglichen Autor(en) und die Quelle ordnungsgemäß nennen, einen Link zur Creative Commons Lizenz beifügen und angeben, ob Änderungen vorgenommen wurden.

Die in diesem Artikel enthaltenen Bilder und sonstiges Drittmaterial unterliegen ebenfalls der genannten Creative Commons Lizenz, sofern sich aus der Abbildungslegende nichts anderes ergibt. Sofern das betreffende Material nicht unter der genannten Creative Commons Lizenz steht und die betreffende Handlung nicht nach gesetzlichen Vorschriften erlaubt ist, ist für die oben aufgeführten Weiterverwendungen des Materials die Einwilligung des jeweiligen Rechteinhabers einzuholen.

Weitere Details zur Lizenz entnehmen Sie bitte der Lizenzinformation auf http://creativecommons.org/licenses/by/4. $0 /$ deed.de.

\section{Literatur}

1. Fraunhofer IEE (2017) IMOWENProjektseite. https:/ / www. iee.fraunhofer.de/de/projekte/suche/laufende/imowen. html.Zugegriffen: 15. Juni 2021

2. (2018) EU-SysFlexProjektwebsite.https:// eu-sysflex.com/ documents/.Zugegriffen: 15. Juni 2021

3. Buhr J, Hänchen H, Kreutziger M, Krahmer S et al (2018) ProjektabschlussberichtSysDL2.0., Dresden

4. (2014) 10-Punkte-Programm der 110-kV-Verteilnetzbetreiber und des Übertragungsnetzbetreibers der Regelzone 50Hertz. https://www.50hertz.com/xspProxy/ Api/StaticFiles/newsxsp/50hertz_flux/dokumente/10_ punkte_programm_systemsicherheit-langfassung.pdf. Zugegriffen: 10. Juni 2021

5. SysDl 2.0 Projektwebsite (2016) Technische Anwendungsszenarien. http://www.sysdl20.de/ergebnisse/. Zugegriffen:20. März2017

6. Kreutziger M, Schegner P, Wende-von-Berg S, Braun M, Bornhorst N (2018) Reactive power management of distributed generators for sleective voltage optimization in 110$\mathrm{kV}$-subtransmission grids. NEIS2018, Hamburg

7. Wende-von Berg S, Bornhorst N et al (2016) SYSDL2.0-Systemdienstleistungen aus Flächenverteilnetzen: Methoden und Anwendungen. 14. Symposium Energieinnovation, Graz

8. Wende-von Berg S, Requardt B et al (2018) Providing ancillary services from distribution grids under the usage of distributed renewable generation: Results from a field test. CIGRE2018, Paris

9. IEC 61970-CGMES:2020 Energy management system application program interface (EMS-API)-Common Grid Model Exchange Specification (CGMES)

10. Verband der Elektrotechnik Elektronik Informationstechnik e. V. (2015) Technische Bedingungen für den Anschluss und Betrieb von Kundenanalagen in das Hochspannungsnetz. VDE-AR-N4120. Verband der Elektrotechnik Elektronik Informationstechnike. V., Berlin

11. Kreutziger M, Becker W, Schegner P, Habermann E (2016) Anwendungsfall-optimierte Bereitstellung von Blindleistung aus dezentralen Erzeugungsanlagen im 110-kV-Verteilnetz. 14. Symposium Energieinnovation, Graz 
12. Fraunhofer IEE (2021) pandapower - Dokumentation. https://pandapower.readthedocs.io/en/v2.6.0/index. html.Zugegriffen:23. Mai2021

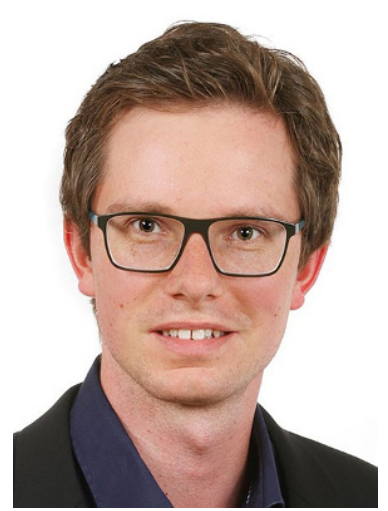

Marcus Kreutziger, wurde 1987 in Karl-Marx-Stadt, Deutschland geboren. Nach Abschluss des Elektrotechnik Studiums an der TU Chemnitz, arbeitet er seit 2013 als wissenschaftlicher Mitarbeiter an der Professur für Elektroenergieversorgung der TU Dresden. Seine Forschungsschwerpunkte sind die Gestaltung von Betriebsführungskonzepten unter Einbeziehung moderner Kommunikationstechnologien sowie den Beiträgen dezentralen Erzeugungsanlagen zu Systemdienstleistungen.

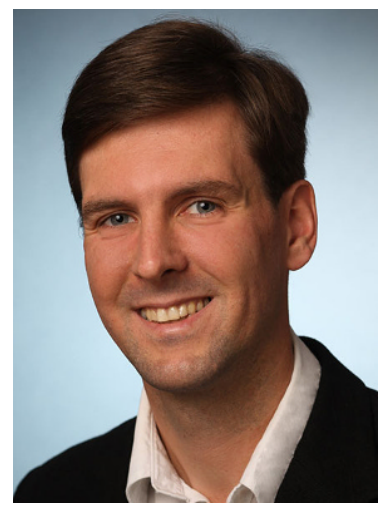

Sebastian Wende-von-Berg, hat ursprünglich Physik studiert und auch in diesem Bereich promoviert (2010). Seit 2015 ist er am Fraunhofer IEE (damals IWES) im Bereich Netzplanung und Netzbetrieb tätig. Seit 2018 ist er Gruppenleiter an der Universität Kassel und seit 2019 Gruppenleiter am IEE. Seine Haupttätigkeitsfelder sind der operative Netzbetrieb sowie Echtzeitsysteme und Anwendungen künstlicher Intelligenz im Kontext der Netzbetriebsführung.

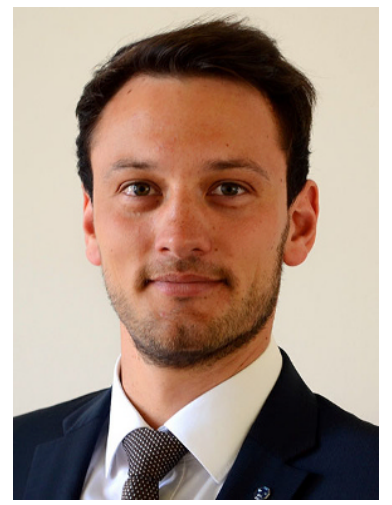

Sebastian Krahmer, studierte Elektrotechnik an der TU Dresden und schloss im Jahr 2014 das Studium mit dem Diplom ab. Seit 2015 ist er als wissenschaftlicher Mitarbeiter am „Institut für Elektrische Energieversorgung und Hochspannungstechnik“ der TU Dresden beschäftigt, wo er seit 2019 Gruppenleiter der Arbeitsgruppe Planung und Betrieb von Netzen ist.

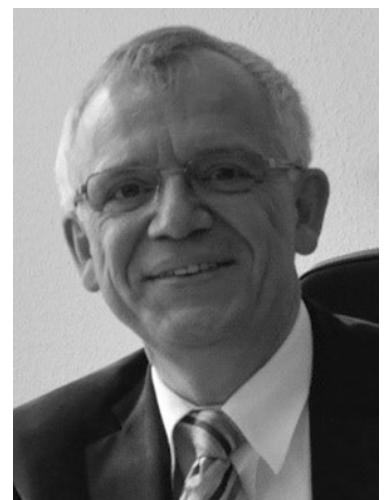

Peter Schegner, ist Professor an der Technischen Universität Dresden und zurzeit Direktor des Institutes für Elektrische Energieversorgung und Hochspannungstechnik. Herr Prof. Schegner leitet zahlreiche Forschungsprojekte, auf den Gebieten: Planung und Betrieb elektrischer Netze, Versorgungsqualität, Entwurf und Betrieb vonSmart Grids, Selektivschutzund Automatisierungstechnik und Stabilität elektrischer Netze. 